\title{
Correction to: EPAC Negatively Regulates Myelination via Controlling Proliferation of Oligodendrocyte Precursor Cells
}

\author{
Zheng-Zheng Gao ${ }^{1} \cdot{\text { Ying-Cong } \mathrm{Li}^{2}}^{2}$ Chong-Yu Shao ${ }^{2} \cdot \mathrm{Jian} \mathrm{Xiao}^{1} \cdot$ \\ Ying Shen ${ }^{2}$ (1) Liang Zhou $^{2,3}$
}

Published online: 7 May 2020

(c) Shanghai Institutes for Biological Sciences, CAS 2020

\section{Correction to: Neurosci. Bull.}

https://doi.org/10.1007/s12264-020-00495-6

The original version of this article unfortunately contained a mistake. The name of first author, Zhen-Zhen Gao, was wrong. It should be Zheng-Zheng Gao.

The original article can be found online at https:// doi.org/10.1007/s12264-020-00495-6.

Ying Shen

yshen@zju.edu.cn

$\bowtie$ Liang Zhou

zllzlj@zju.edu.cn

1 Molecular Pharmacology Research Center, School of Pharmaceutical Sciences, Wenzhou Medical University, Wenzhou 325035, Zhejiang, China

2 Department of Neurobiology, Zhejiang University School of Medicine, Hangzhou 310058, China

3 Key Laboratory of Brain Science, Guizhou Institution of Higher Education, Zunyi Medical University, Zunyi 563000, China 